However, this may result from the low prevalence of haemoproteus: less than 6 per cent of cocks had the parasite. The small population size and isolation of the Mono County leks probably explains the absence of serious parasites there.

Several other studies apparently show that parasite-free males are not the preferred mating partners. A particularly clear example was a three-year study of grey tree frogs (Hyla versicolor). No consistent negative correlation was observed between call duration, the primary cue in female choice, and helminth or nematode infestation (G. Hausfater, University of Missouri, Columbia). But this does not refute the parasite hypothesis because none of these parasites had more than minor physiological effects on tree frogs. There is little or no benefit for a female picking a mate free of parasites that are, in general, non-debilitating and non-lethal.

One area of the parasite hypothesis that remains untested is the claim that heritable variation in parasite resistance is maintained by coevolutionary cycles between host and parasite genotypes. But heritable resistance has been reported in ring-necked pheasants (Phasanius colchicus; see figure). Chicks were reared either under hygienic conditions, being fed low levels of an anti-protozoan drug (coccidia being the main parasite) or under unhygienic, untreated conditions to allow full exposure to parasites (N. Hillgarth, University of Oxford). Mortality in the untreated group was twice as high as in the treated group, but offspring of untreated parents survived better than those of treated parents. Having been subject to the rigours of parasite selection, the untreated parents ought to have higher natural resistance, which they did.

Andrew Pomiankowski is in the Department of Zoology, University of Oxford, South Parks Road, Oxford OX1 3PS, UK.

1. Hamilton, W.D. \& Zuk, M. Science 218, 384-388 (1982) 2. Pomiankowski, A.N. Oxford Sun. Evol. Biol. 7, 136 (1988)

3. Read, A. Trends Ecol. Evol. 3, 97-102 (1988)

4. Maynard Smith, J. J. theor. Biol. 115, 1-8 (1985)

5. Andersson, M. Evolution 40, 804-816 (1986).

\title{
THERMOMETRY
}

\section{Ironing out the standard scale}

\section{R. L. Rusby}

From 1 January 1990 the boiling point of water at standard pressure will no longer be taken to be $100^{\circ} \mathrm{C}$ exactly. For many people this may be the most striking consequence of the decision, taken recently by the International Committee for Weights and Measures, to revise the International Temperature Scale. The new scale will incorporate many advances in both fundamental and practical thermometry which have been made since the last revision in 1968.

Since 1954, the kelvin has been defined by assigning the value $273.16 \mathrm{~K}$ to the temperature of the triple point of water the unique point at which ice, water and water vapour co-exist in equilibrium. Celsius temperatures have been defined by subtracting 273.15 from the kelvin equivalents. It follows from these definitions that the values of all other temperatures, the boiling point of water included,

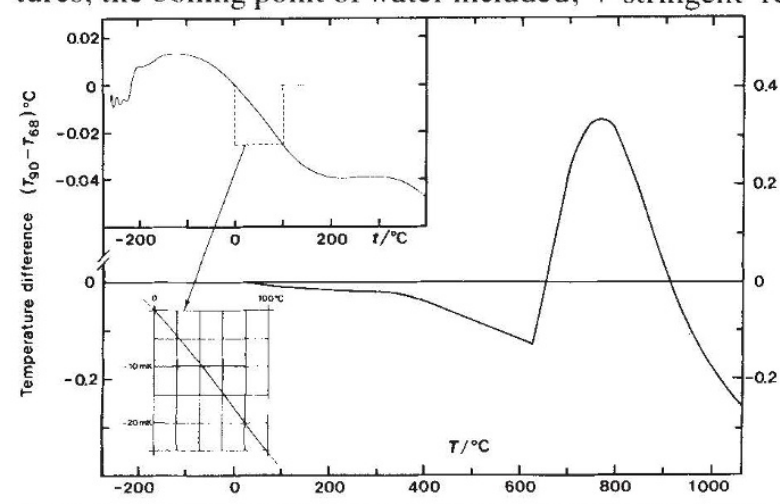

Approximate differences between temperatures on the 1990 and 1968 scales must be determined using thermodynamic instruments such as the constant-volume gas thermometer. This is a laborious process: as Callender noted in 1899: "it is impossible for those who have never worked with a gas thermometer to realise the extent of its shortcomings", and no practitioner today would disagree.

On the other hand there are many devices which respond to temperature changes with good precision and reproducibility, and these can be used as practical thermometers even if the values obtained are not accurate in the thermodynamic sense. The objective of the International Temperature Scale, of which Callendar was a pioneer, has always been to combine this precision and reproducibility with as good thermodynamic accuracy as can be achieved, and so provide a scale to satisfy all but the most stringent requirements. The first International Temperature Scale was adopted in 1927, and revisions were undertaken in 1948 and 1968 to improve its accuracy, to introduce more reproducible fixed points. and to extend the temperature range. The new scale, to be known as ITS-90, is a continuation of this process.

For the first time primary techniques other than gas thermometry have contributed to the realignment of temperature values. Thus values above $500{ }^{\circ} \mathrm{C}$ will be based principally on spectral radiation pyrometry using Planck's radiation law. Some corroborative data have been obtained from measurements of the electrical ('Johnson') noise in a resistor, and at the National Physical Laboratory, J. E. Martin, T. J. Quinn and B. Chu (Metrologia 25, 107$112 ; 1988)$, have used a total radiation calorimeter relying on the StefanBoltzmann $T^{4}$ law to produce new data between $-130{ }^{\circ} \mathrm{C}$ and $+100{ }^{\circ} \mathrm{C}$. This independent method confirms the results of gas thermometry carried out at the National Bureau of Standards (NBS; now the National Institute of Standards and Technology) near Washington DC, which was the first to suggest that the 1968 scale was substantially in error (L. A. Guildner \& R. E. Edsinger J. Res. natn. Bur. Stand. A80, 703-738; 1976). For example, these experiments indicate a value for the 'steam point' of $99.975^{\circ} \mathrm{C}$, unexpectedly (five standard deviations) different from its conventional value of $100^{\circ} \mathrm{C}$.

ITS-90 will implement many other improvements which will have more practical effect than the numerical changes. Boiling points will be replaced by the more reproducible freezing or triple points wherever possible (the steam point itself will not be included), and the mathematical formulation will be revised to reduce errors of interpolation. The range will be extended down to $0.65 \mathrm{~K}$ by including helium vapour pressure equations - but the millikelvin physicist will continue to plough a lonely furrow for some time to come.

Perhaps the most significant development, and the most difficult to accomplish. has been the removal of the platinumrhodium thermocouple which is used in the 1968 scale above $630^{\circ} \mathrm{C}$. The thermocouple is responsible for the erratic errors in that range, as shown in the figure, and it is not sufficiently reproducible for presentday standards. Its replacement has required the development of platinum resistance thermometers capable of operating with good stability up to the freezing point of silver, about $962{ }^{\circ} \mathrm{C}$. Beyond this point ITS-90 will use radiation pyrometry, which is currently specified only above the freezing point of gold, which is at a temperature of near $1,064^{\circ} \mathrm{C}$.

With the introduction of the ITS-90, we shall for the first time have a coherent scale extending from below $1 \mathrm{~K}$ up to the freezing point of gold and beyond, based on instruments of proven reproducibility. The ultimate goal of a practical thermodynamic thermometer remains elusive, but the new scale should serve as the basis of improved temperature standards for a good many years.

R. L. Rusby is in the Division of Quantum Metrology, National Physics Laboratory. Teddington TW11 OLW, UK. 